\title{
Т.В. МАЙСТРОВИЧ
}

\section{Теоретические основы разработки национального стандарта «Библиотечно-информационные услуги научной библиотеки. Виды, формы и режимы предоставления»}

Реферат. Статья содержит обоснование теоретических положений, положенных в основу национального стандарта ГОСТ Р 7.0.104-2019 «СИБИД. Библиотечно-информационные услуги научной библиотеки. Виды, формы и режимы предоставления». Подтверждается целесообразность применения кластерного подхода, позволившего подойти к библиотечно-информационной услуге с точки зрения ее содержания (вида), формы и режимов предоставления пользователям. Показывается возможность применения стандарта не только для научных, но и для библиотек других типов. Определение библиотечно-информационной услуги в стандарте дает возможность понимать ее как конкретный результат библиотечного обслуживания и информационной деятельности библиотеки. Стандартом определены пять видов услуг: библиотечные, библиографические, информационные, библиометрические, консультационные. Часть из них является общими для всех типов библиотек, но библиометрические составляют специфику библиотек научных. Каждый вид реализуется в той или иной форме, под которой в стандарте понимается способ предоставления библиотечно-информационной услуги в рамках существующей в научной библиотеке организации библиотечно-информационного обслуживания.

Формы библиотечной услуги: выдача документа во временное пользование; передача его содержания путем копирования; предоставление возможности ознакомления с документами. Библиографические услуги реализуются в форме сообщения, содержащего справку или библиографическую консультацию по запросу; библиографического списка публикаций и библиографической продукции. В научных библиотеках составляются тематические или отраслевые указатели, списки публикаций отдельных лиц и коллективов, списки с ссылками на труды заданных лиц и коллективов. К библиографическим услугам отнесены повышение библиографической грамотности, обучение пользователей созданию библиографической записи, формированию библиографического аппарата научных и учебных работ. Формами информационной услуги признаны предоставление пользователям информационных продуктов и полнотекстовой информации, отобранной и систематизированной в соответствии с определенными критериями.

Прерогативой преимущественно академических и вузовских библиотек являются библиометрические услуги, при этом формы их оказания достаточно разнообразны и зависят от степени приближенности научной библиотеки непосредственно к исследовательскому процессу и

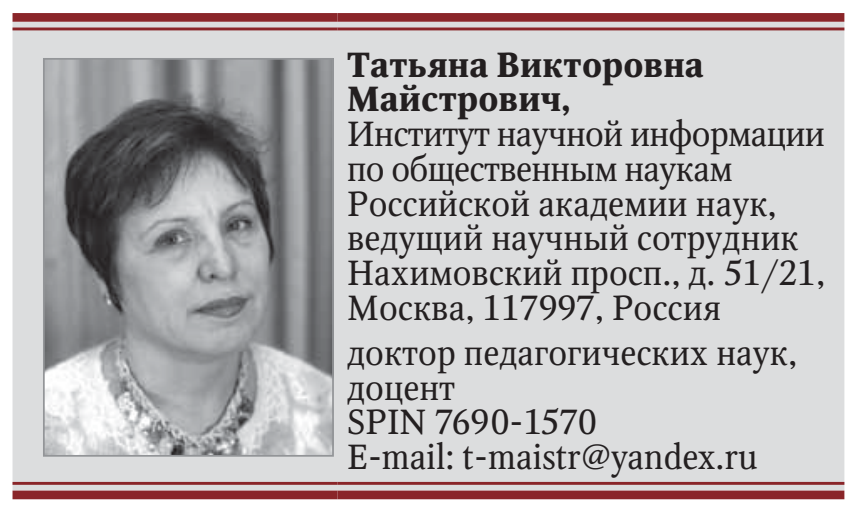


распределения работы в структуре НИИ. В стандарте устанавливаются следующие библиометрические услуги: предоставление пользователю формализованных показателей эффективности научной работы, создание аналитического продукта на основе библиометрических и наукометрических исследований, проверка научных работ на наличие некорректного заимствования. Консультационные услуги оказываются в большинстве библиотек, но в научных добавляется консультирование по оформлению научных работ, по нормативным и организационно-распорядительным документам, представлению результатов собственной научной деятельности.

В стандарт включены не только формы услуг, но и параметры, конкретизирующие их оказание: периодичность, причина оказания, адресность, экономические характеристики, место оказания. Еще один контур стандарта - режим предоставления и получения услуги, который не влияет на ее сущность, но может скорректировать ее востребованность. Режимы характеризуются степенью самостоятельности работы пользователя, синхронизацией заказа, выполнения и получения услуги, периодичностью обслуживания, средствами и каналом коммуникации.

В статье уделяется внимание сервисному подходу к библиотечно-информационным услугам. В промежуточную версию стандарта был включен раздел «Сервисные опции» (о способах повышения комфортности получения пользователем библиотечно-информационной услуги, исходя из его индивидуальных предпочтений), снятый в окончательной редакции из-за недостаточной проработанности проблемы.

В заключение отмечается, что ГОСТ позволяет приблизить обслуживание ученых к их реальным потребностям и запросам. В качестве перспективы называется разработка стандарта, определяющего обязательную номенклатуру услуг для библиотек каждого типа и вида, а также критериев их качественной оценки.

Ключевые слова: библиотечно-информационная услуга, виды информационно-библиотечных услуг, библиографическая услуга, библиотечная услуга, информационная услуга, библиометрическая услуга, консультационная услуга, режим предоставления информационно-библиотечной услуги, научная библиотека, библиотечно-информационное обслуживание.

Для цитирования: Майстрович Т.В. Теоретические основы разработки национального стандарта «Библиотечно-информационные услуги научной библиотеки. Виды, формы и режимы предоставления» // Библиотековедение. 2019. Т. 68, № 5. С. 465-474. DOI: 10.25281/0869-608X-2019-685-465-474.

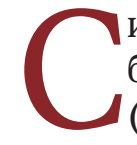

истема стандартов по информации, библиотечному и издательскому делу (СИБИД) до недавнего времени имела существенные диспропорции с точки зрения отражения библиотечной практики [1]. В сфере библиотечного обслуживания действовали два стандарта: ГОСТ 7.0-99 «СИБИД. Информационно-библиотечная деятельность, библиография. Термины и определения» [2] и ГОСТ Р 7.0.20-2014 «СИБИД. Библиотечная статистика. Показатели и единицы исчисления» [3]. Но в 2018-2019 гг. были утверждены сразу два национальных стандарта: терминологический ГОСТ Р 7.0.103-2018 «СИБИД. Библиотечно-информационное обслуживание. Термины и определения» [4], разработанный под руководством Российской государственной библиотеки, и технологический ГОСТ Р 7.0.104-2019 «СИБИД. Библиотечно-информационные услуги научной библиотеки. Виды, формы и режимы предоставления» [5], который является первым целенаправленно раскрывающим сущность библиотечно-информационных услуг, их содержание и формы предоставления, головной разработчик - Институт научной информации по общественным наукам (ИНИОН) PAH.

\section{Содержание и целевое назначение Стандарта «СИБИД. Библиотечно- информационные услуги научной библиотеки. Виды, формы и режимы предоставления ”}

Задачей национального стандарта ГОСТ Р 7.0.104-2019 «СИБИД. Библиотечноинформационные услуги научной библиотеки. Виды, формы и режимы предоставления» является определение основных видов библиотечноинформационных услуг, раскрытие их содержа- 
ния, форм предоставления и режимов «доставки» пользователям. ГОСТ адресован, в первую очередь, научным библиотекам и органам научнотехнической информации (НТИ), исходя из их типологических характеристик, основанных на целеполагании - библиотечно-информационной поддержке научной деятельности.

Специфику работы научной библиотеки определяют такие факторы, как однородность потребителей (образовательный уровень, тематика запросов и т. д.), стабильность их запросов, совместное (с библиотекой или органом НТИ) определение форм и режимов оказания услуг, комфортность и своевременность получения услуги. Важно и то, что библиотечно-информационные услуги, оказываемые научными библиотеками и органами НТИ (далее - научная библиотека), направлены на обеспечение деятельности и отдельного ученого, и научного коллектива, и научно-исследовательского (научно-образовательного) учреждения. Например, для научных библиотек есть необходимость обслуживания ученого на его рабочем месте, которым, в том числе, является персональный компьютер. В связи с этим одной из основ рассматриваемого стандарта является концепция «распределенного рабочего места» ученого (в лаборатории, издательстве, дома, в командировке и т. п.), изложенная Л.И. Госиной [6] и О.И. Левченко [7], в соответствии с которой услуга должна быть оказана в той форме и том режиме, которые определены пользователем. Однако данный ГОСТ, несмотря на указанную адресность, вполне может быть использован в библиотеках других типов и видов в силу заложенных в нем теоретических и методических положений, позволивших применить кластерный подход к библиотечно-информационным услугам.

В настоящее время мы не имеем общепризнанной классификации библиотечно-информационных услуг и даже их единого наименования. Например, ГОСТ 7.0-99 приводит три взаимосвязанных термина:

- информационная услуга: предоставление информации определенного вида потребителю по его запросу;

- библиотечная услуга: конкретный результат библиотечного обслуживания, удовлетворяющий определенную потребность пользователя библиотеки (выдачу и абонирование документов, предоставление информации о новых поступлениях, справки, выставки, консультации и т. д.);
- библиографическая услуга: результат библиографического обслуживания, удовлетворяющий потребности в библиографической информации [2].

Нетрудно заметить рассогласование методик формирования этих определений: услуга в одном случае - это действие (предоставление), в двух других - результат.

ГОСТ Р 7.0.20-2014 объединил это разнообразие в одном термине: библиотечно-информационная услуга - «конкретный результат библиотечного обслуживания, удовлетворяющий определенную потребность пользователя библиотеки (выдача документов, предоставление информации о новых поступлениях, справки, выставки, консультации и т. д.)» [3]. Стандарт ГОСТ Р 7.0.103-2018 «Библиотечно-информационное обслуживание. Термины и определения» использует этот же термин с практически идентичным определением [4].

Разумеется, терминология ГОСТ Р 7.0.1042019 находится в полном соответствии с профильными терминологическими стандартами, в частности с ГОСТ Р 7.0.103-2018. Однако указанная в последнем синонимия между библиотечно-информационной и библиотечной услугами (п. 3.1.2) оказалась мало приемлемой для практики, поскольку библиотечная услуга является одной из форм (наряду с другими) библиотечно-информационных услуг, что и нашло отражение в ГОСТе.

Кроме того, важно было ответить на вопрос, все ли услуги, которые оказываются или могут оказываться в стенах библиотеки правомерно отнести к сущностным, присущим библиотеке как учреждению, имеющему определенные (базовые) социальные функции, связанные с организацией общественного использования документов [8]. Задачи библиотеки изложены в документе, определяющем правомерность ее функционирования (устав, положение и др.). Библиотека может оказывать иные содержательные услуги, но если они не являются результатом профильной деятельности, то не могут быть отнесены к библиотечно-информационным, не относятся к ним офисные и технические услуги. Исходя их этого в ГОСТ Р 7.0.104-2019 библиотечно-информационная услуга понимается как конкретный результат библиотечного обслуживания и информационной деятельности библиотеки, предусмотренных ее уставом или иным регламентирующим документом, предлагаемый пользователю в соответствии с его запросом или ожиданием [5, п. 4.2]. 
При разработке стандарта возникла необходимость во введении и обосновании некоторых терминов, не встречающихся ранее в системе СИБИД, например:

- библиометрическая услуга: предоставление данных, полученных на основе изучения профильного потока публикаций посредством библиометрического анализа;

- каналы предоставления библиотечноинформационной услуги: технико-технологические способы доставки библиотечно-информационной услуги пользователям;

- режим оказания библиотечно-информационной услуги: совокупность временных, дистанционных, технических и технологических факторов, определяющих способы и каналы доставки/предоставления конкретной формы услуги пользователю.

Методологической основой послужила работа В.В. Брежневой и В.А. Минкиной, в которой авторы «разложили» услугу поэлементно, включив ее «внешнее оформление» [10].

Научные библиотеки оказывают пользователям пять видов библиотечно-информационных услуг: библиографические, библиометрические, библиотечные, информационные, консультационные. Каждый вид реализуется в той или иной форме, под которой понимается способ оказания библиотечно-информационной услуги в рамках существующей в библиотеке организации библиотечно-информационного обслуживания.

Библиотечные услуги связаны с предоставлением документов, являющихся объектами библиотечного хранения. По своему содержанию эта услуга является документной, поскольку оперирует именно документами, тем не менее разработчики стандарта пришли к согласованному решению остановиться на привычном наименовании.

Библиотечные услуги и формы их оказания практически универсальны для библиотек и заключаются в предоставлении документов во временное пользование из фонда библиотеки, фондов других библиотек (книговыдача); предоставлении текста документа (как аналогового, так и электронного) в постоянное пользование (путем его легитимного копирования); ознакомлении пользователя с документом, например, путем организации выставок. В стандарте в качестве отдельной формы указано наличие доступа (в том числе дистанционного) к полнотекстовым электронным ресурсам собственной генерации и полу- чаемым на основе лицензионных соглашений. Однако, строго говоря, эта услуга может иметь своим результатом как предоставление документа во временное пользование («только для чтения»), так и в постоянное пользование (если разрешено копирование). В данном случае разработчики стандарта пожертвовали теоретической «чистотой» ради удобства практического применения.

Библиографические и информационные услуги могут заключаться в подготовке информационного/библиографического продукта любого объема либо предварительно, по решению библиотекаря (каталог - для облегчения книговыдачи или подготовка библиографического издания), либо по запросу пользователя (тематический список литературы). Предоставление информационного продукта (продукции) в качестве услуги обосновано И.С. Пилко [11], но нам в данном случае особенно важно, что сам библиотечный продукт может рассматриваться и в качестве услуги, если он создается в ответ на конкретный запрос, на что обратила внимание В.В. Брежнева [12, с. 15-16]. Во-вторых, пользователь получает недокументированный результат интеллектуального труда сотрудников, которым является устная справка или консультация.

Одним из дискуссионных вопросов при разработке стандарта стало разграничение библиографических и информационных услуг. Необходимо отметить, что объем понятия «информационная услуга» гораздо шире библиотечной и библиографической деятельности. Основным назначением справочных бюро, различных информационных центров является оказание информационной услуги. Но это один из тех случаев, когда мы имеем исходную неопределенность терминологии, а в задачу технологического стандарта не входит решение столь сложных задач. Тем не менее остается фактом существенная близость и самого содержания этих видов услуг, и форм их реализации.

Библиографические услуги реализуются в форме сообщения, содержащего справку или библиографическую консультацию по запросу пользователя; библиографического списка публикаций; библиографической продукции. В научных библиотеках помимо тематических или отраслевых указателей составляются списки публикаций отдельных лиц и коллективов, а также списки, где имеются ссылки на труды заданных лиц и коллективов. К библиографи- 
ческим услугам отнесены повышение библиографической (библиотечно-информационной) грамотности, обучение пользователей (проведение занятий и консультаций по созданию библиографической записи, формированию библиографического аппарата научных и учебных работ - пристатейной и/или прикнижной библиографии).

Формами информационной услуги признаны предоставление пользователям информационных продуктов (любого объема, содержащих библиографическую, фактографическую информацию или их комбинацию, подготовленных научной библиотекой или полученных из других источников в постоянное или временное пользование) и полнотекстовой информации, отобранной и систематизированной в соответствии с критериями, сформулированными в запросе пользователя.

Научно-вспомогательные услуги (библиометрические, информетрические и наукометрические), приобретают огромное значение для оценки деятельности ученого, научного коллектива и научного учреждения. Прерогативой преимущественно академических и вузовских библиотек является библиометрический анализ публикаций сотрудников соответствующего научного или образовательного учреждения с целью выявления эффективности работы каждого из них и института в целом согласно принятым в наукометрии индексам. Формы библиометрических и наукометрических услуг достаточно разнообразны и зависят от степени приближенности научной библиотеки непосредственно к исследовательскому процессу, т. е. они особенно важны для библиотек и органов НТИ научно-исследовательских учреждений. В стандарте устанавливаются следующие библиометрические услуги: предоставление пользователю формализованных показателей и рейтингов, принятых для оценки качества научной работы; индивидуальный мониторинг рейтингов цитируемости ученых; создание аналитического продукта на основе библиометрических и наукометрических исследований научного направления; проверка подготовленных или полученных научных работ на наличие некорректного заимствования. Безусловно, этот перечень может быть расширен в соответствии с реальными условиями работы научной библиотеки, распоряжением руководителя НИИ, сложившимся разделением труда между библиотекой, органом НТИ и аппаратом ученого секретаря.
Консультационные услуги оказываются в большинстве библиотек (консультации по пользованию библиотекой, ее справочно-поисковым аппаратом, электронными ресурсами различной генерации и т. д.) но в научных библиотеках имеют свою специфику, выраженную в консультировании по оформлению научных работ; по нормативным и организационно-распорядительным документам, необходимым для осуществления научной, образовательной, научно-организационной деятельности; по представлению результатов собственной научной деятельности (подача заявок на патентование, депонирование и т. д.).

Библиотечно-информационная услуга оказывается не только в определенных формах, но и имеет конкретизацию по различным параметрам. Наиболее значимыми из них (как для пользователя, так и для организации обслуживания) являются:

- периодичность оказания: разовые (по запросу) и постоянные (по заказу или договору);

- причина оказания: по запросу пользователя, без запроса (инициативные со стороны библиотеки);

- адресность: индивидуальные (для конкретного потребителя), групповые, коллективные, массовые;

- экономические характеристики: бесплатные для конечного пользователя, платные;

- место оказания: стационарные, внестационарные.

Очевидно, что вне зависимости от формы предоставления библиотечно-информационная услуга должна быть «доставлена» пользователю, т. е. иметь еще один технологический контур - режим получения услуги. Практически любая библиотечно-информационная услуга может осуществляться в различном режиме, который не влияет на ее сущность, но может скорректировать ее востребованность.

Режимы предоставления и получения библиотечно-информационных услуг подразделяются по следующим параметрам:

- степень самостоятельности работы пользователя: режим самообслуживания и «клиентский» режим (услуга, оказываемая персоналом научной библиотеки); в режиме самообслуживания осуществляется отбор документов в открытом доступе, использование электронной библиотеки, иных электронных ресурсов и баз данных, в том числе электронных каталогов, заказ документов из хранилищ, копирование текстов и др. (к услугам, оказываемым персо- 
налом научной библиотеки, относят, например, выдачу документов или их копий);

- синхронизация заказа, выполнения и получения услуги: режим реального времени, режим отложенного обслуживания, упреждающий режим;

- периодичность обслуживания: разовый («запрос-ответ»), постоянный, продолжающийся (передача информации по мере ее накопления), текущий (обслуживание с определенной заранее оговоренной периодичностью);

- средства коммуникации: устные и письменные услуги;

- каналы коммуникации: «контактный» (в стандарте услуги, предоставляемые в помещении библиотеки, названы локальными) и дистанционный режимы; в этом смысле термин «электронная услуга» $[4$, п. 3.1.2.1] может использоваться как краткое обозначение услуги, оказанной в дистанционном режиме на основе компьютера (но не телефона, например).

Такой детальный подход к режимам оказания услуг имеет большое методическое значение для библиотек. В частности, благодаря этому удалось достичь понимания (в том числе руководящих органов), что услуга, оказанная посредством компьютера, не превращает ее в виртуальную, тем более не делает виртуальным пользователя библиотеки (которым чаще всего является сотрудник какого-либо НИИ). На самом деле пользователю оказана обычная услуга, но в дистанционном режиме. Практическая значимость данного подхода проявляется, например, в достижении большей адекватности учета библиотечно-информационных услуг научной библиотеки. Для этого необходимо принимать во внимание, что учетной единицей является форма услуги, а режимы ее оказания самостоятельного значения для определения объема работы не имеют (это не отрицает возможность дифференцированного дополнительного учета и по режимам). Например, непосредственное посещение пользователем научной библиотеки не может рассматриваться как единственное основание (учетная единица) услуги, поскольку это только один из режимов ее оказания.

\section{Сервис в контексте библиотечно-информационных услуг}

В проекте рассматриваемого нами стандарта присутствовал раздел «Сервисные опции для библиотечно-информационных услуг, предоставляемых научной библиотекой» (при полном понимании возникшей тавтологии, что непреодолимо в рамках технологического стандарта). Понятие сервисных услуг активно разрабатывается библиотековедами [13; 14, с. 19; 15]. В.В. Брежнева в целом относит библиотечное дело к сервисной деятельности [12, с. 15-16]. В число сервисных услуг включаются возможность напечатать и переплести подготовленные документы; перевод, библиографическое редактирование списка к работам; доставка книг на дом; продление сроков пользования книгами; получение справки по телефону; комплекс услуг, увеличивающих комфортность библиотечного обслуживания (индивидуальный абонемент, МБА, ММБА, нестационарные формы обслуживания, дифференцированное обслуживание абонентов в помещении библиотеки) и др. И.Г. Юдина и О.Л. Лаврик указывают, что сервисные услуги - «это услуги, предоставляемые с использованием различной компьютерной, аудиовизуальной и другой техники (предоставление читателю компьютера для работы, ксерокопирование, сканирование и т. п.). При выполнении этого вида услуг не происходит дополнительной интеллектуальной работы с первичным документом» [16, с. 56].

Но проанализировав перечисляемые в публикациях формы сервисных услуг, мы должны признать, что это не самостоятельные услуги. Сервис в научных библиотеках направлен на создание для пользователей дополнительных удобств в получении документных, информационных и научно-вспомогательных услуг. И это позволяет говорить не о сервисных услугах, а о сервисных опциях, улучшающих качество получения той или иной информационно-библиотечной услуги. Сервисные опции библиотечно-информационной услуги - это способы повышения комфортности ее получения, исходя из индивидуальных предпочтений пользователя. Они могут прилагаться к любой библиотечно-информационной услуге и определяются материально-техническими возможностями научной библиотеки, спецификой запросов. При этом наличие сервисных опций ни в коем случае не означает перевод библиотечно-информационной услуги на платную основу. К сервисным опциям можно отнести технические (аренда компьютера, если пользователь забыл свой); транспортные (доставка документов на дом); предоставление лицензионных ресурсов на персональные устройства пользователя и т. д. 
Однако результаты профессионального обсуждения показали недостаточную проработанность такого подхода, Тем не менее очевидно, что любая библиотечно-информационная услуга может получать дополнительные свойства, повышающие ее комфортность для пользователя. Дополнительные свойства могут касаться формы услуги, сроков ее оказания, способов доставки пользователю (список не исчерпывающий). Скорее всего, со временем эта тема получит углубленную проработку, что позволит сделать библиотечно-информационное обслуживание более индивидуализированным.

ГОСТ Р 7.0.104-2019 «СИБИД. Библиотечно-информационные услуги научной библиотеки. Виды, формы и режимы предоставления» позволяет приблизить обслуживание ученых к их реальным потребностям, исходя из того, что «оптимальная модель обслуживания ученых в библиотеке НИИ должна базироваться на том, чтобы пользователь, не отвлекаясь от основного процесса - научных исследований, затрачивал как можно меньше времени на поиск необходимых ресурсов и имел перед глазами единое документно-информационное пространство, организованное по профильному признаку. Для ученого должны быть созданы все условия для самообслуживания. Развитие ИБО должно быть направлено на приближение информационнобиблиотечной среды к пользователю, в отличие от существующей в настоящее время необходимости адаптации пользователя к ней» [7, с. 12].

В целом для библиотечной практики этот ГОСТ полезен тем, что на его основе могут быть разработаны методические пособия, позволяющие улучшить качество оказания библиотечно-информационных услуг и более корректно подойти к их учету.

Хотелось бы надеяться, что данное направление стандартизации будет иметь дальнейшее развитие, поскольку очевидно, что при общности основных видов услуг номенклатура и формы их оказания в библиотеках различного типа и вида отличаются существенным образом. Например, для детских библиотек важен спектр зрелищных и развивающих услуг (библиотечные театрализованные вечера, организация праздников, экскурсий, кружков, мероприятия по развитию навыков чтения и т. д.). Следовательно, можно говорить о целесообразности подготовки стандарта, определяющего обязательную номенклатуру услуг для библиотек каждого типа и вида, а так же критериев их качественной оценки.

\section{Список источников}

1. Майстрович Т.В. Национальная стандартизация // Независимый библиотечный адвокат. 2015. №1. C. $3-10$.

2. ГОСТ 7.0-99. Система стандартов по информации, библиотечному и издательскому делу. Информационно-библиотечная деятельность, библиография. Термины и определения // Библиотечное дело : государственные и национальные стандарты / Российская государственная библиотека. Москва : Пашков дом, 2014. С. 15-31.

3. ГОСТ Р 7.0.20-2014. Система стандартов по информации, библиотечному и издательскому делу. Библиотечная статистика. Показатели и единицы исчисления. Москва: Стандартинформ, 2014. 37 c.

4. ГОСТ Р 7.0.103-2018. Система стандартов по информации, библиотечному и издательскому делу. Библиотечно-информационное обслуживание. Термины и определения. Москва : Стандартинформ, 2018. 30 c.

5. ГОСТ Р 7.0.104-2019. Система стандартов по информации, библиотечному и издательскому делу. Библиотечно-информационные услуги научной библиотеки. Виды, формы и режимы предоставления. Москва : Стандартинформ, 2019.7 с.

6. Госина Л.И. Библиотечное обслуживание ученых на рабочем месте: концепция развития системы услуг в отраслевой библиотеке РАН // Новые технологии в информационном обеспечении науки : сб. науч. тр. Москва : Биоинформсервис, 2001. С. 68-76.

7. Левченко О.И. Информационно-библиотечное обеспечение пользователей в научно-исследовательских институтах РАН естественнонаучного профиля : автореф. дисс. ... канд. пед. наук. Москва, 2014. 26 с.

8. Майстрович Т.В. Библиотека в социальном институте чтения // Библиосфера. 2015. № 1. С. $58-62$.

9. Номенклатура информационных услуг : метод. пособие. Москва : ИПКИР, 1982.78 с.

10. Брежнева В.В., Минкина В.А. Информационное обслуживание: продукты и услуги, предоставляемые библиотеками и службами информации предприятий. 2-е изд., перераб. Санкт-Петербург : Профессия, 2008. 304 с.

11. Пилко И.С. Информационные и библиотечные технологии : учеб. пособие. Санкт-Петербург : Профессия, 2006. 501 с.

12. Брежнева В.В. Информационное обслуживание: концепция сервисного развития : автореф. дис. ... докт. пед. наук. Санкт-Петербург, 2007. 42 с. 
13. Дворкина М.Я. Библиотечное обслуживание: новая реальность : лекции. Москва : Изд-во МГУКИ : Профиздат, 2002. 44, [3] с.

14. Обеспечение качества информационно-библиотечного обслуживания : пособие для руководителей библиотек / РБА ; отв. сост. и рук. проекта Куликова Л.В. ; сост. Кузнецова Т.В. и др. Санкт-Петербург : Изд-во РНБ, 2013. 174 с.
15. Алешин Л.И., Ордынская М.А. Библиотечные сервисы : науч.-практ. пособие. Москва : Литера, 2015. 303 c.

16. Юдина И.Г., Лаврик О.Л. Информационная функция в теории и практике библиотечного дела / Сиб. отд-ние Рос. акад. наук, Гос. публич. науч.техн. б-ка ; науч. ред. Б.С. Елепов. Новосибирск, 2010. 228 c.

\title{
Theoretical Basis for the
}

Development of National Standard "Library and Information Services of the Scientific Library. Types, Forms and Modes of Provision"

Tatiana V. Maystrovich, Institute of Scientific Information for Social Sciences of the Russian Academy of Sciences, 51/21 Nakhimovsky Prospekt, Moscow, 117997, Russia SPIN 7690-1570

E-mail: t-maistr@yandex.ru

\begin{abstract}
The article presents substantiation of the theoretical provisions underlying the National Standard GOST R 7.0.104-2019 "SIBID. Library and information services of the scientific library. Types, forms and modes of provision”. The author proves the expediency of applying cluster approach, allowed to consider the library and information service in terms of its content (type), form and modes of provision to users. The article demonstrates possibility of applying Standard not only to scientific libraries, but also to the libraries of other types. Definition of library and information service in the standard makes it possible to understand it as a specific result of library services and information activities of the library. The standard determines five types of services: library, bibliographic, information, bibliometric, consulting. Some of them are common to all types of libraries, but bibliometric services are specific feature of scientific libraries. Each type of services is implemented in one form or another, under which the standard refers to the method of providing library and information services in the framework of the existing scientific library organization of library and information services.

Forms of library services: loan of a document for temporary use; transfer of its contents by copying; providing the possibility to familiarize with documents. Bibliographic services are implemented in the form of message containing reference or bibliographic advice on request, bibliographic list of publications and bibliographic products. Scientific libraries compose thematic or subject field indexes, lists of publications of individuals and collectives, lists with references to the works of specified persons and collectives. Bibliographic services include improving bibliographic literacy, training of users to create a bibliographic record, the formation of bibliographic apparatus of scientific and educational works. The recognized forms of information services are providing users with information products and full-text information, selected and systematized in accordance with the certain criteria. The prerogative of mainly academic and university libraries are bibliometric services, while the forms of their provision are quite
\end{abstract}


diverse and depend on the degree of proximity of the scientific library directly to the research process and distribution of work in the structure of the research Institute. The standard establishes the following bibliometric services: providing user with formalized performance indicators of scientific work, creation of analytical product based on bibliometric and scientometric studies, checking of scientific works for incorrect borrowing. Consulting services are available in most libraries, but in scientific libraries there is added scientific advice on the design of scientific papers, normative, regulatory and administrative documents, presentation of results of individual research activities. The module includes not only the forms of services, but also the parameters specifying their provision: frequency, reason for rendering, targeting, economic characteristics, service location. Another contour of the module is the mode of providing and receiving services, which does not affect its essence, but may adjust its demand. Modes are characterized by the degree of independence of the user, synchronization of the order, execution and receipt of services, frequency of service, means and channel of communication. The article focuses on the service approach to library and information services. The intermediate version of the standard included the section "Service options" (on the ways to improve the comfort of obtaining library and information services by the user, based on his individual preferences), removed in the final version due to insufficient elaboration of the problem. In conclusion, the author notes that National Standard allows bring the service of scientists to their real needs and requests. As a perspective, the paper calls the development of Standard defining the mandatory range of services for libraries of each type and kind, as well as criteria for their qualitative assessment.

Key words: library and information service, types of information and library services, bibliographic service, library service, information service, bibliometric service, consulting service, mode of provision of information and library services, scientific library, library and information services.

Citation: Maystrovich T.V. Theoretical Basis for the Development of National Standard "Library and Information Services of the Scientific Library. Types, Forms and Modes of Provision”, Bibliotekovedenie [Library and Information Science (Russia)], 2019, vol. 68, no. 5, pp. 465-474. DOI: 10.25281/0869-608X2019-68-5-465-474.

\section{References}

1. Maistrovich T.V. National Standardization, Nezavisimyi bibliotechnyi advokat [Independent Library Lawyer], 2015, no. 1, pp. 3-10 (in Russ.).

2. State Standard 7.0-99. System of Standards on Information, Librarianship and Publishing. Information and Librarian Activity, Bibliography. Terms and Definitions, Bibliotechnoe delo: gosudarstvennye $i$ natsional'nye standarty [Librarianship: State and National Standards]. Moscow, Pashkov Dom Publ., 2014, pp. 15-31 (in Russ.).

3. GOST R 7.0.20-2014. Sistema standartov po informatsii, bibliotechnomu i izdatel'skomu delu. Bibliotechnaya statistika. Pokazateli i edinitsy ischisleniya [State Standard R 7.0.20-2014. System of Standards on Information, Librarianship and Publishing. Library Statistics. Indicators and Numeration Units]. Moscow, Standartinform Publ., 2014, 37 p.

4. GOST R 7.0.103-2018. Sistema standartov po informatsii, bibliotechnomu i izdatel'skomu delu. Bibliotechnoinformatsionnoe obsluzhivanie. Terminy i opredeleniya [State Standard R 7.0.103-2018. System of Standards on Information, Librarianship and Publishing. Library and Information Service. Terms and Definitions]. Moscow, Standartinform Publ., 2018, 30 p.

5. GOST R 7.0. 104-2019. Sistema standartov po informatsii, bibliotechnomu i izdatel'skomu delu. Bibliotechno-informatsionnye uslugi nauchnoi biblioteki. Vidy, formy i rezhimy predostavleniya [State Standard $\mathrm{R}$ 7.0. 104-2019. System of Standards on Information, Librarianship and Publishing. Library and Information Services of Scientific Libraries. Types, Forms and Modes of Provision]. Moscow, Standartinform Publ., 2019, 7 p.

6. Gosina L.I. Workplace Library Services for Scientists: A Concept of Service System Development in the RAS Branch Library, Novye tekhnologii v informatsionnom obespechenii nauki: sb. nauch. tr. [New Technologies in Information Support of Science: collected scientific papers]. Moscow, Bioinformservis Publ., 2001, pp. 68-76 (in Russ.).

7. Levchenko O.I. Informatsionno-bibliotechnoe obespechenie pol'zovatelei v nauchno-issledovatel'skikh institutakh RAN estestvennonauchnogo profilya [Information and Library Support of Users in Natural Sci- 
ence Institutes of the Russian Academy of Sciences], cand. ped. sci. diss. abstr. Moscow, 2014, 26 p.

8. Maistrovich T.V. Library in the Social Institution of Reading, Bibliosfera [Bibliosphere], 2015, no. 1, pp. 58-62 (in Russ.).

9. Nomenklatura informatsionnykh uslug: metod. posobie [Nomenclature of Information Services: methodological manual]. Moscow, IPKIR Publ., 1982, 78 p.

10. Brezhneva V.V., Minkina V.A. Informatsionnoe obsluzhivanie: produkty i uslugi, predostavlyaemye bibliotekami i sluzhbami informatsii predpriyatii [Information Service: Products and Services Provided by Enterprise Libraries and Information Centers]. St. Petersburg, Professiya Publ., 2008, 304 p.

11. Pilko I.S. Informatsionnye i bibliotechnye tekhnologii: ucheb. posobie [Information and Library Technologies: textbook]. St. Petersburg, Professiya Publ., 2006, 501 p.

12. Brezhneva V.V. Informatsionnoe obsluzhivanie: kontseptsiya servisnogo razvitiya [Information Service:
Service Development Concept], doct. ped. sci. diss. abstr. St. Petersburg, 2007, 42 p.

13. Dvorkina M.Ya. Bibliotechnoe obsluzhivanie: novaya real'nost': lektsii [Library Service: A New Reality: lectures]. Moscow, MGUKI Publ., Profizdat Publ., 2002, $44 \mathrm{p}$.

14. Kulikova L.V. (ed.) Obespechenie kachestva informatsionno-bibliotechnogo obsluzhivaniya: posobie dlya rukovoditelei bibliotek [Ensuring the Quality of Information and Library Services: guide for library managers]. St. Petersburg, RNB Publ., 2013, 174 p.

15. Aleshin L.I., Ordynskaya M.A. Bibliotechnye servisy: nauch.-prakt. posobie [Library Services: scientific and practical guide]. Moscow, Litera Publ., 2015, $303 \mathrm{p}$.

16. Yudina I.G., Lavrik O.L. Informatsionnaya funktsiya $v$ teorii i praktike bibliotechnogo dela [Information Function in the Theory and Practice of Librarianship]. Novosibirsk, 2010, 228 p.

\section{Анонс}

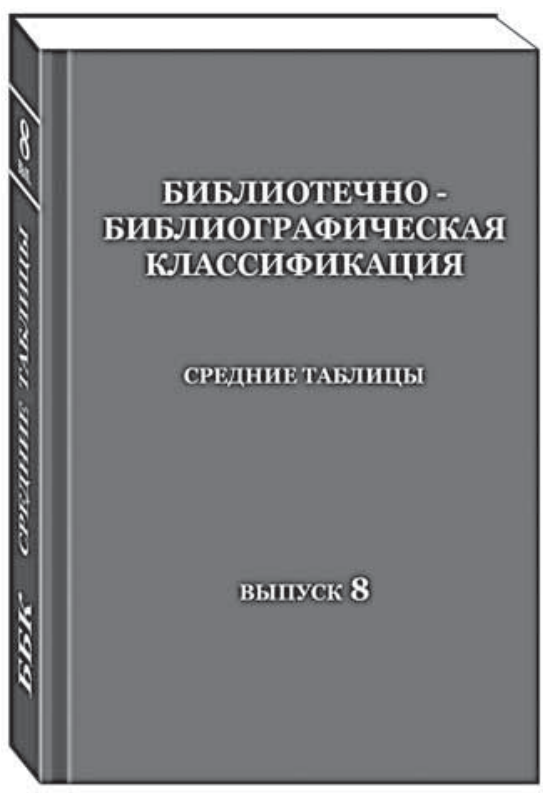

Библиотечно-библиографическая классификация : Средние таблицы : практическое пособие / Рос. гос. б-ка, Рос. нац. б-ка, Б-ка Российской акад. наук. Москва: Пашков дом, 2019.

Вып. 8. 1 А Междисциплинарное знание. 9 Я Литература универсального содержания. Типовые деления общего применения. $456 \mathrm{c.}$

Средние таблицы ББК предназначены для систематизации литературы в универсальных научных библиотеках, библиотеках высших учебных заведений, в отраслевых и специализированных научных библиотеках, для использования в работе центральных библиотек ЦБС и для учебных заведений, готовящих библиотечные кадры.

Восьмой выпуск объединяет таблицы нового отдела классификации «1 Междисциплинарное знание», дополненные и исправленные таблицы отдела «9 Литература универсального содержания» и новую редакцию таблиц Типовых делений общего применения.

\section{Справки и заказ изданий:}

119019, Москва, ул. Воздвиженка, д. 3/5

Российская государственная библиотека,

Издательство «Пашков дом», отдел книжных изданий Pashkov_Dom@rsl.ru,Pashkov_Dom.Book@rsl.ru http://store.rsl.ru/service/pashkov_dom +7 (499) 557-04-70, доб. 25-72 